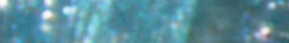

\title{
Análisis y metodología científica aplicados a la obra textil de Mariano Fortuny y Madrazo
}

\author{
Elena González Arteaga, Maria Antonia García Rodriguez
}

Resúmen: En este artículo presentamos la metodología analítica y los resultados obtenidos en el laboratorio de materiales del Instituto de Patrimonio Cultural de España (IPCE) para la identificación de materiales presentes en diversas obras de Mariano Fortuny pertenecientes al Museo del Traje de Madrid (CIPE). Han sido seleccionadas un total de 15 piezas, divididas en dos grupos pertenecientes a indumentarias y textiles. Entre ellas se encuentran tres colgaduras, ocho piezas de distintas indumentarias, tres colgaduras y cuatro tejidos planos. Los análisis realizados pretenden alcanzar los siguientes objetivos: en primer lugar determinar la técnica de elaboración, en segundo lugar explicar la aplicación del color en los estampados de determinadas piezas textiles.

Palabras clave: Fortuny, análisis de tejidos, indumentaria, colgaduras, tejidos planos, Delphos, colorantes

\section{Analysis and scientific methodology applied to the textile work of Mariano Fortuny and Madrazo}

\begin{abstract}
This article presents the analytical methodology and results in the identification in the Institute of Cultural Heritage of Spain (IPCE) materials laboratory of materials in some textile works by Mariano Fortuny belonging to the Costume Museum of Madrid (CIPE). A total of 15 pieces have been selected, divided into two groups, those belonging to clothing and textiles. Among them are three wall hangings, eight pieces from different clothing, three wall hangings and four planes tissues. Analyzes performed are intended to achieve the following objectives: firstly determining the technical development and secondly explain the application of color in the prints of other textiles.
\end{abstract}

Keyword: Fortuny, analysis of textile garments, clothing, wall hangings, planes tissues, Delphos, dyes

\section{Análises e metodología científica aplicados à obra textil de Mariano Fortuny e Madrazo}

Resumo: Neste artigo apresentamos a metodologia analítica e os resultados obtidos no laboratório do IPCE na identificação dos materiais presentes em diversas obras de Mariano Fortuny, pertencentes ao Museo del Traje de Madrid (CIPE). Foram selecionadas um total de 15 peças, divididas em dois grupos respeitantes a indumentárias e têxteis. Entre elas encontram-se três colgaduras, oito peças de distintas indumentárias, três colgaduras e quatro tecidos planos. As análises realizadas pretendem alcançar os seguintes objetivos: a) determinar a técnica de elaboração, b) explicar a aplicação da cor nos estampados de determinadas peças têxteis.

Palavras-chave: Fortuny, análises de tecidos, indumentária, colgaduras, tecidos planos, Delphos, corantes 


\section{Introducción}

Es habitual encontrar el calificativo de "mago" en relación a Mariano Fortuny y atribuirle poderes en la obtención de colores para sus pinturas o en el tintado de telas en sus obras. Fortuny tenía control total sobre cada proceso de producción y tal vez esto es lo que ha conducido a un desconocimiento en cuanto a los materiales que utilizaba y en la creación de leyendas en la historia de la moda como la obtención del extraordinario plisado ondulado del Delphos. Asimismo, Fortuny creaba colores de matices insospechados, una paleta de tonalidades propia de un pintor. [VV.A.A, 2010; V.V.A.A, 2017]

Nacido en Granada, y criado en París, Mariano Fortuny y Madrazo (1981-1949) pasó su vida adulta en Venecia y su carrera abarcó más de cincuenta años. Descendiente de dos de las familias más ilustres del siglo XIX, su padre era el famoso pintor Mariano Fortuny y Marsal y su madre Cecilia de Madrazo y Garreta procedía de la célebre saga de los Madrazo, con destacados nombres como José, Federico, Raimundo y Ricardo de Madrazo. Aunque su padre falleció tempranamente a los 36 años su influencia marcó su trayectoria y animado tanto por su madre como por su tío Raimundo Madrazo, con tan solo siete años Mariano comenzó a pintar. En este ambiente se gestó su amor por la pintura, los viajes, el coleccionismo de objetos curiosos, el orientalismo, los tejidos y la tecnología. Fue artista, pintor, grabador, fotógrafo, escenógrafo y pionero diseñador textil además de un apasionado de la ciencia estudiando óptica y electricidad. En su faceta como inventor fue destacable su contribución en las artes escénicas, patentando un nuevo sistema de iluminación escénica y en 1904 la denominada Cúpula Fortuny, un ciclorama situado en la parte posterior del escenario que servía como pantalla y que explotaba todas las posibilidades de la luz indirecta. También creó otros muchos ingenios, hasta completar un total de cincuenta patentes. Las múltiples incursiones en todos estos ámbitos artísticos y científicos de Fortuny explican su genialidad creadora y dan coherencia a su polifacética carrera.

Fortuny estaba bien considerado como artista en una amplia gama de géneros, pero fue a través de sus diseños de ropa y textiles donde sus sensibilidades artísticas excepcionales alcanzaron su cenit, así como una gran audiencia internacional. En 1906 tiene lugar su primera aparición como diseñador, revolucionando la encorsetada moda femenina de la época creando una toga denominada Knossos, éxito entre las grandes bailarinas como Isadora Duncan, Eleonora Duse o Sarah Bernhardt. En 1907 crea el vestido Delphos, de túnica de satén de seda plisada que patentó en 1909 (patente no 414119 Genre d'etoffe plisée ondulée) y que con los años se convertiría en su seña de identidad. Sus modelos contribuirían a cambiar la moda y la moral de los primeros años del s.XX [De Osma: 1994].

Los acabados y la textura de los diseños textiles e indumentarias producidos en exclusiva en el Palacio Pesaro Orfei forman parte del misterio que le rodea y, aunque para
Mariano Fortuny la tela siempre fue el punto de partida, nunca fabricó los tejidos, aún poseyendo un profundo conocimiento sobre ellos. Utilizó diversos materiales como algodón, lino, seda y lana, empleando uno o varios de estos materiales en una misma pieza [Llorente, 2005].

Con las técnicas analíticas actuales intentaremos dar respuesta a algunas incógnitas sobre la elaboración de sus diseños, realizados dentro del más absoluto secretismo por Fortuny y su mujer Henriette. Estas técnicas proveen una valiosa información sobre los materiales constitutivos de la obra, su naturaleza, estructura y composición, donde la metodología utilizada dependerá de la técnica analítica que se elija, lo que requiere de muchos aspectos a tener en cuenta.

Entre las técnicas utilizadas en la Sección de Análisis de Materiales del Instituto del Patrimonio Cultural de España (IPCE) se encuentran la microscopía electrónica de barrido - microanálisis por dispersión de energías de rayos $X$ (SEM-EDX), la cromatografía líquida de alta resolución (HPLC), la cromatografía en capa fina (TLC) y diversos test microquímicos que darán respuesta a algunas de las cuestiones planteadas sobre las obras de Mariano Fortuny.

\section{Metodología}

La identificación de los materiales constitutivos de un tejido puede dar respuesta a una serie de preguntas relacionadas con las técnicas de ejecución, la época y lugar de manufactura, así como con aspectos sobre la degradación de los mismos y la detección de intervenciones anteriores. La metodología general de trabajo seguida en el Área de Investigación y Formación-Sección de Análisis de Materiales del IPCE se puede resumir en las siguientes etapas: a) estudio in situ de la obra; b) toma de muestra y etiquetado de las mismas; c) examen bajo el microscopio estereoscópico; f) tratamiento previo de las muestras antes de la realización de los análisis según la técnica elegida; g) aplicación de las diferentes técnicas de análisis [González et ali, 2010].

La elección de la técnica analítica más adecuada se ha realizado dependiendo de la información que se necesite para la caracterización de la pieza textil y de la cantidad de muestra disponible. Las muestras se han tomado en zonas no visibles o de menor interés artístico de la pieza y con la mínima cantidad necesaria para el estudio, siempre manteniendo un alto valor representativo.

La identificación de la seda o algodón en las piezas seleccionadas de Mariano Fortuny se efectuó mediante la sección longitudinal y transversal de la fibra. En el análisis de la sección longitudinal las fibras se separaron lo más unitariamente posible sobre un portaobjetos de vidrio donde se añadió el reactivo idóneo y al igual que las estratigrafías de las secciones transversales se observaron al microscopio. El microscopio utilizado es un Olimpus BX51, provisto de luz reflejada y polarizada e iluminación UV. 
Para la caracterización de los materiales utilizados en la técnica de estampación como polvos metálicos o pigmentos inorgánicos, se empleó la técnica de microscopía electrónica de barrido - microanálisis por dispersión de rayos X (SEMEDX). Consiste en el microanálisis de las preparaciones estratigráficas o de las muestras depositadas en un stub de microscopía. El equipo es un Oxford Link Pentafet, acoplado a un microscopio electrónico de barrido Jeol-5800.

Pigmentos como el azul de Prusia de algunos tejidos planos o distintos adhesivos acrílicos encontrados en colgaduras e indumentarias se identificaron mediante la técnica de espectroscopia de infrarrojos por transformada de Fourier FTIR con un equipo Bruker -Equinox 55 [García et ali, 2008].

La cromatografía líquida de alta resolución (HPLC) con un cromatógrafo Waters $660 \mathrm{E}$ para la separación de los aminoácidos presentes en las proteínas nos permitió identificar huevo en algunas de las muestras estudiadas. Se realiza en primer lugar un tratamiento previo de la muestra con una hidrólisis y posterior derivatización. El cromatograma se comparó con patrones de los que dispone el IPCE identificando de esta forma las proteínas presentes.

La mayoría de los tintes contienen más de un componente responsable del color, puesto que son elaborados a menudo con mezclas de compuestos o combinaciones de distintos tintes. De todas las técnicas cromatográficas, las más empleadas para el análisis de colorantes son la cromatografía en capa fina o TLC y la cromatografía liquida de alta resolución o HPLC ya que son capaces de separar los compuestos de mezclas complejas [Ferreira, 2004].

La identificación de los tintes como el de cochinilla se efectuó por cromatografía en capa fina. Para realizar este análisis cromatográfico es necesario, en primer lugar, liberar el tinte de la fibra mediante un tratamiento de muestra que incluye varias etapas como hidrólisis, extracción, purificación y redisolución del colorante. Los análisis se realizaron siempre en paralelo con patrones de colorantes naturales que dispone el IPCE. También se hicieron ensayos de coloración como el de almidón o fuschina y un micro test para la evaluación de la presencia o ausencia de indigotina propuesto por J. Hofenk de Graaff. [Hofenk de Graaff, 2004]. Este test microquímico es sencillo, rápido y fiable y aunque sólo se puede aplicar en la determinación de un componente ha resultado muy útil en la identificación del tinte de índigo o hierba pastel en trabajos anteriores [Arteaga et ali, 2010].

\section{Descripción de las piezas}

Todas las piezas pertenecen al Museo del Traje de Madrid (CIPE) y se dividen en dos grandes grupos (indumentarias y textiles). Los resultados obtenidos para cada una de las piezas se explican de forma detallada en los apartados posteriores.

Se han estudiado un total de ocho piezas de indumentaria, dentro de las cuales se incluyen tres Delphos, dos Vestidos, una Chaqueta de Teatro, una Túnica y una Dalmática. Los denominados Delphos son una especie de túnica de satén de seda plisada cuya estructura los hacía apropiados para viajar, ya que ocupaban poco espacio y no necesitaban plancha.

Los textiles se dividen en dos grupos, tejidos planos y colgaduras, analizándosecuatroytres piezas respectivamente.

A continuación se da una breve descripción de cada pieza en base al catálogo del Museo del Traje de Madrid (CIPE).

\section{Indumentaria}

- Vestidos.- El número de piezas son tres vestidos Delphos cuya técnica de elaboración es el plisado, un vestido realizado con la técnica de Gasa Marquisette y el vestido Eleonora con un fondo de terciopelo negro y una exquisita decoración floral.

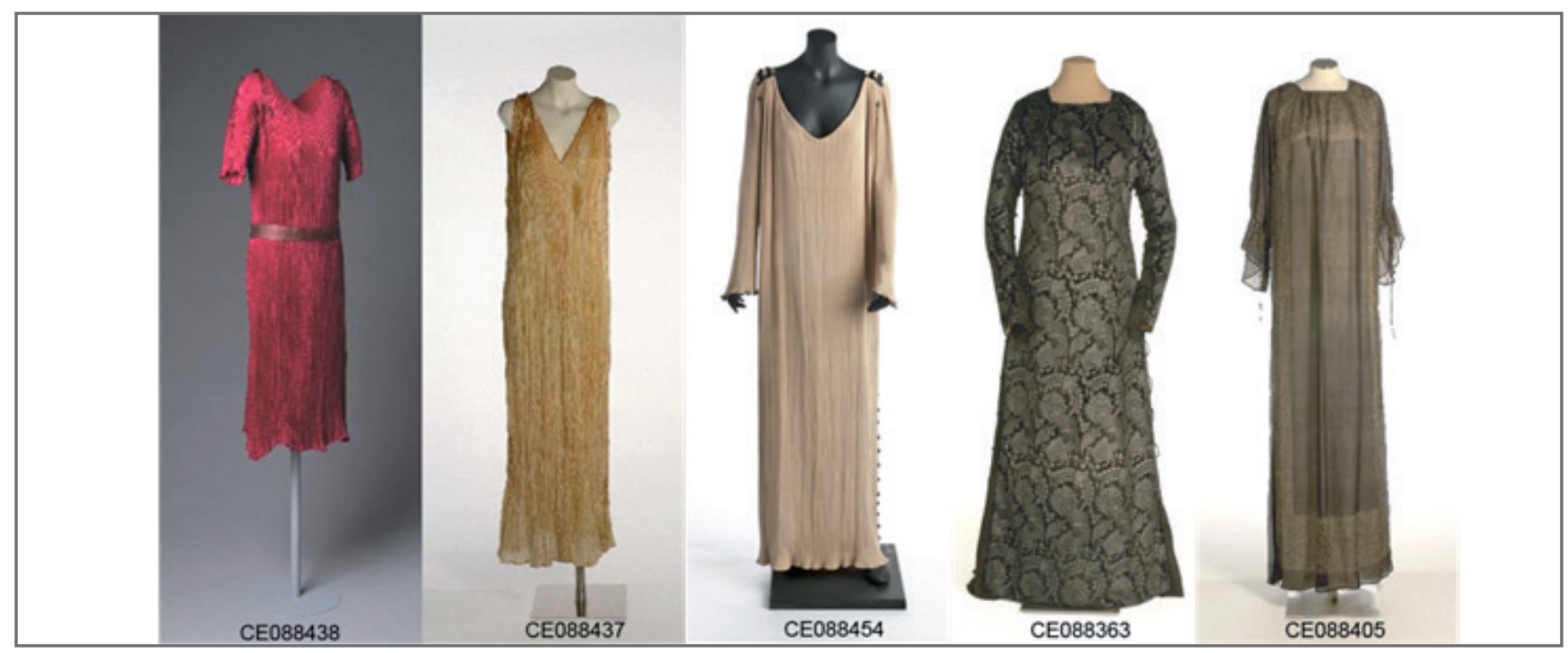

Figura 1.- Vestidos estudiados. http://ceres.mcu.es/. Fotos piezas: Munio Rodil; Lucía Ybarra; Francisco Javier Maza 
Todos los vestidos están realizados en seda. En el caso de los Delphos las coloraciones van del púrpura, al amarilloy amarillo claro (beige). El otro vestido en Gasa Marquisette de seda negra, repite la forma de los vestidos Eleonora en terciopelo, y al igual que ellos, en los laterales va abierto mostrando una pieza plisada con el mismo sistema que los Delphos. Fortuny juega con el sutil contraste de color entre el negro de la gasa y el dorado en la decoración y forro [figura 1].

-Dalmática.- Esta indumentaria eclesiástica en seda azul de manga corta y con una decoración vegetal de cardo, flores y ramas estarcidas en verde posee dos bandas pardas adornadas con ojivas y vegetales estarcidas en plata, que se colocan verticalmente recorriendo la espalda y el delantero. Estas, en ambos casos, son cortadas perpendicularmente por otras dos horizontales, formando un cuadrado, donde se coloca un fragmento de otro tejido [figura 2].

-Chaqueta de teatro.- Cuerpo hecho con terciopelo en seda negro que lleva una decoración estarcida en dorado. Diseño destinado a la obra de teatro de"Otelo", para la que Fortuny se inspiró en un joven que aparece en el cuadro "La despedida de los Embajadores" de Vittore Carpaccio [figura 2].

-Túnica.-Túnica abierta de seda rosa con motivos geométricos estarcidos en oro [figura 2].

\section{Textiles}

-Tejidos planos.- De perfil rectangular se han seleccionado un total de cuatro. Todos ellos son de lino o algodón, la mayoría con estarcidos y realizados con diferentes técnicas tales como estampación, sarga, terciopelo o tafetán. Los colores de los fondos son diferentes como violetas, pardos o azules y van decorados con motivos vegetales geométricos o animalísticos en azul, verde o pardo [figura 3].

- Colgaduras.- Se analizaron tres realizadas con diferentes materiales. Presentan técnicas habituales como la del tafetán además del estarcido [figura 4].

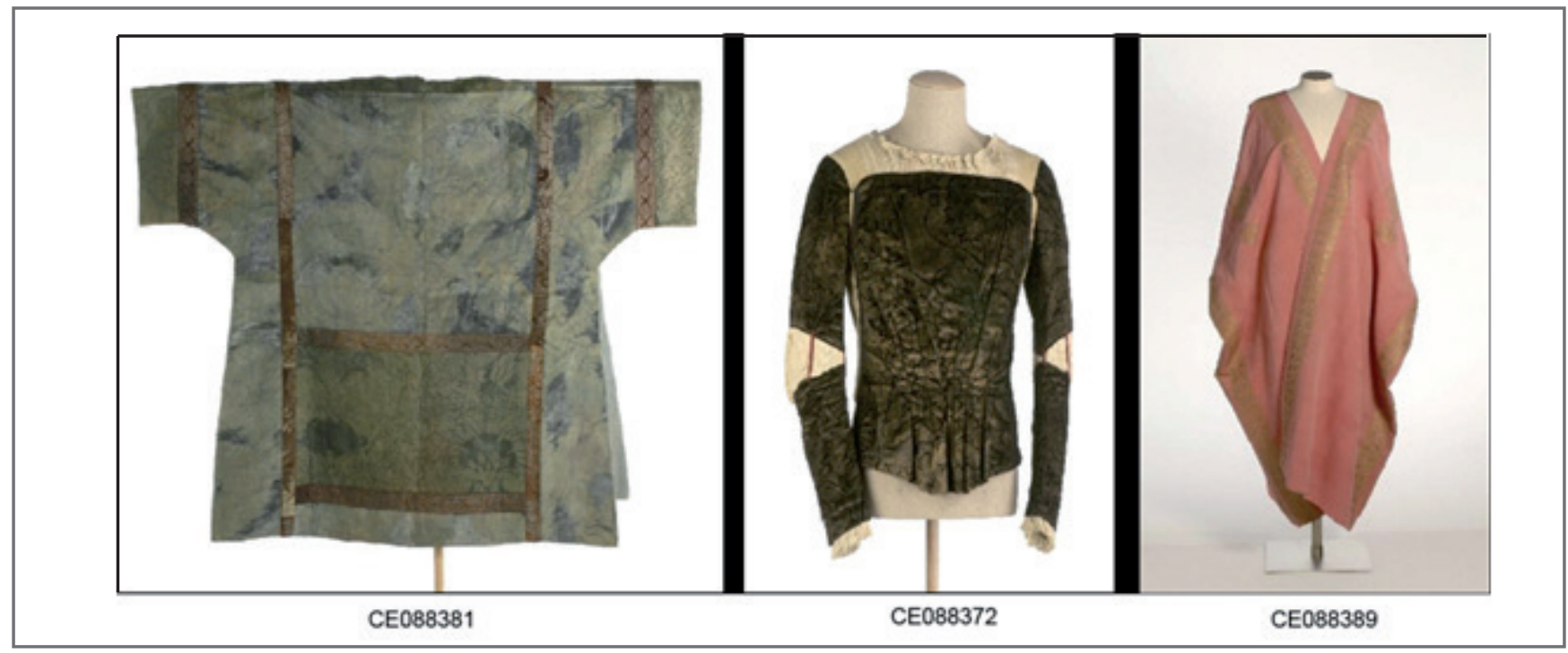

Figura 2.-Dalmática, Chaqueta de teatro y Túnica. http://ceres.mcu.es/. Fotos piezas: Munio Rodil; Lucía Ybarra; Francisco Javier Maza.

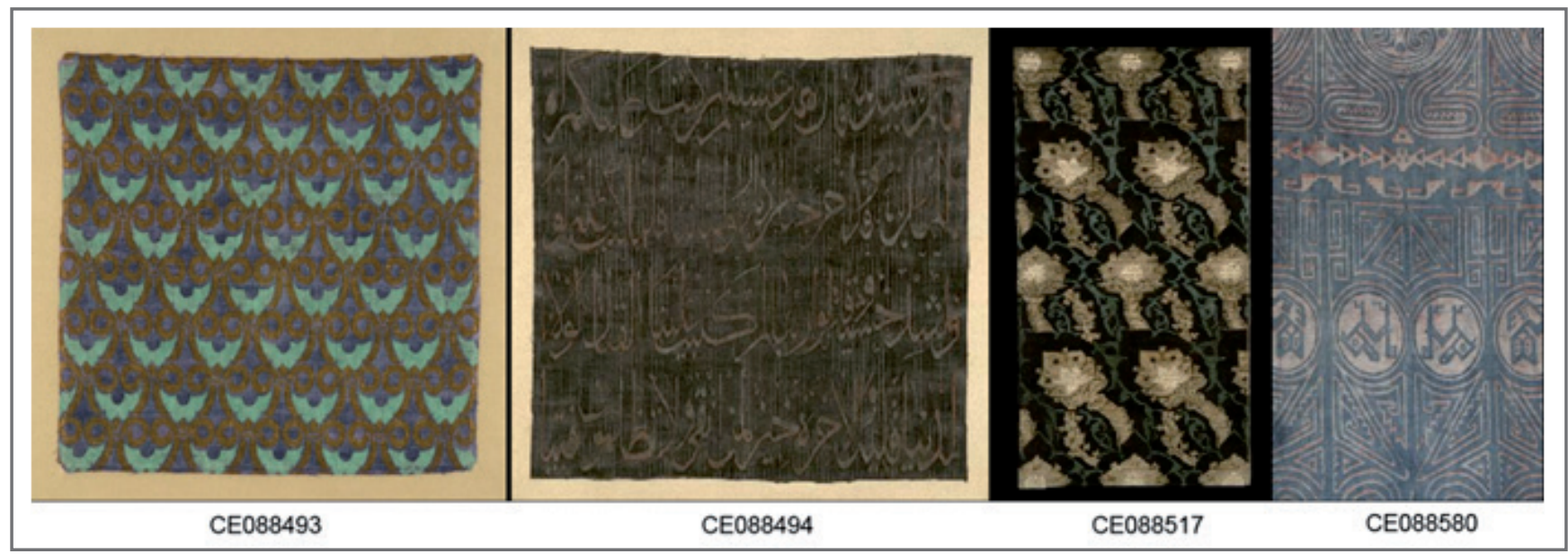

Figura 3.-Tejidos planos estudiados. http://ceres.mcu.es/. Fotos piezas: Munio Rodil; Lucía Ybarra; Francisco Javier Maza. 


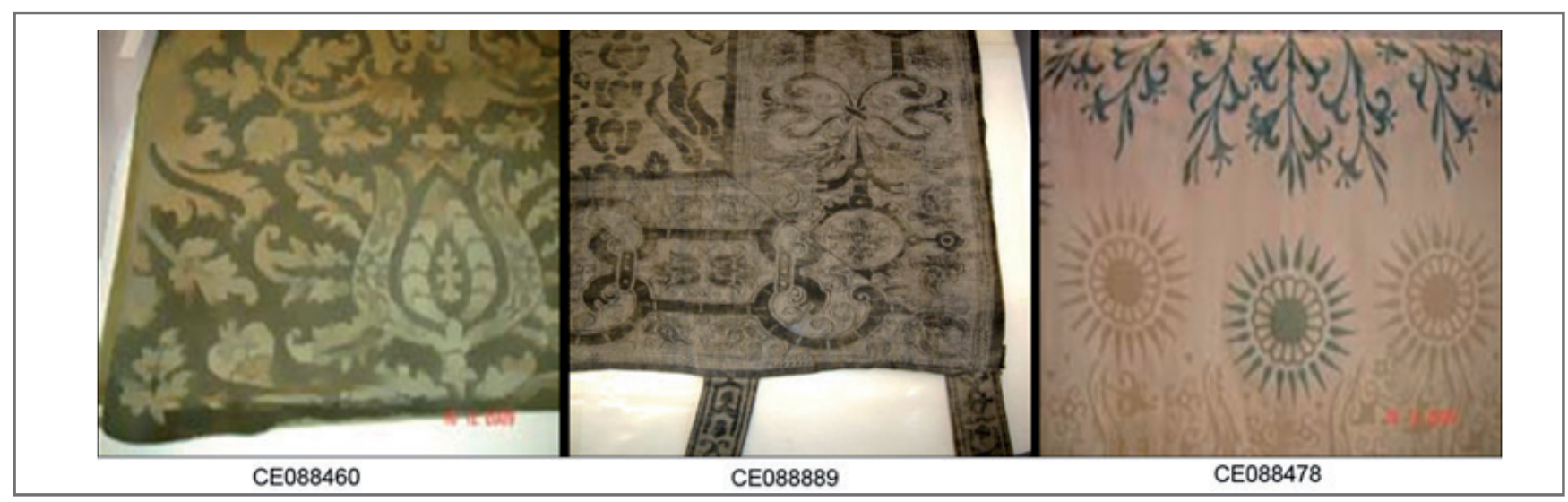

Figura 4.-Colgaduras estudiadas. Fotos piezas: Elena González

\section{Toma de muestras}

El número de muestras analizadas han sido 32 y se presentan en las siguientes tablas.

\begin{tabular}{|c|c|c|c|}
\hline TIPOLOGÍA & $\mathrm{N}^{\circ}$ INVENTARIO & $\begin{array}{c}\mathbf{N}^{\circ} \\
\text { Muestra }\end{array}$ & DESIGNACION \\
\hline \multirow{10}{*}{ INDUMEN'TARIA } & CE088438 (Delphos rojo) & 10 & Zona inferior costura (centro del plisado) \\
\hline & CE088437 (Delphos amarillo) & 11 & Fragmento parte in ferior (reverso parte posterior) \\
\hline & $\begin{array}{c}\text { CE } 088454 \\
\text { (Delphos amarillo claro) }\end{array}$ & 17 & Interior de la costura del lateral derecho, parte inferior \\
\hline & \multirow{2}{*}{ CE088381 (Dalmática) } & 8 & Anverso de la parte delantera de la pieza, esquina inferior izquierda \\
\hline & & 9 & Próximos a la muestra 8 , parte trasera esquina inferior izquierda \\
\hline & $\begin{array}{c}\text { CE088363 } \\
\text { (Vestido Eleonora) }\end{array}$ & 13 & Manga izquierda (para ver el estarcido) \\
\hline & \multirow{2}{*}{ CE088405 (Vestido) } & 12 & Adhesivo del plisado lateral izquierdo \\
\hline & & 14 & Parte derecha del cuello (estarcido de color dorado) \\
\hline & $\begin{array}{c}\text { CE088372 } \\
\text { (Chaqueta de Traje de Teatro) }\end{array}$ & 15 & Anverso de la parte delantera del cuello parte derecha \\
\hline & CE088389 (Túnica) & 16 & Parte posterior izquierda, tomada en el dobladillo \\
\hline
\end{tabular}

\begin{tabular}{|c|c|c|c|}
\hline \multicolumn{4}{|c|}{ Tabla 2.- Tom a de muestras TEJIDOS PLANOS } \\
\hline TIPOLOGÍA & N $^{\circ}$ INVENTARIO & $\begin{array}{c}\mathrm{N}^{\circ} \\
\text { Muestra }\end{array}$ & DESIGNACION \\
\hline \multirow{11}{*}{$\begin{array}{l}\text { TEJIDOS } \\
\text { PLANOS }\end{array}$} & \multirow{2}{*}{ CE088493 } & 18 & Hilo tomado del extremo superior izquierdo (verde y algo de violeta) \\
\hline & & 19 & Hilo dorado extremo in ferior derecho \\
\hline & \multirow{3}{*}{ CE088494 } & 20 & Hilo extremo inferior derecho gris y de color cobrizo \\
\hline & & 21 & Hilo plateado próximo a la muestra 20 \\
\hline & & 22 & Hilo para análisis de fibras, tomado en la misma zona que las anteriores \\
\hline & \multirow{3}{*}{ CE088517 } & 24 & Verde, en el dobladillo externo inferior izquierdo \\
\hline & & 25 & Pardo con dorado en la misma zona del dobladillo que la muestra 24 \\
\hline & & 26 & Negro próximo a la muestra 24 \\
\hline & \multirow{3}{*}{ CE088580 } & 27 & Rojo y azul (hilo), extremo inferior izquierdo \\
\hline & & 28 & Rojo (micro-fragmento) \\
\hline & & 29 & Azul (hilo azul) \\
\hline
\end{tabular}

\begin{tabular}{|c|c|c|c|}
\hline \multicolumn{4}{|c|}{ Tabla 3.- Toma de muestras COLGADURAS } \\
\hline TIPOLOGÍA & $\mathrm{N}^{\circ}$ INVENTARIO & $\begin{array}{c}\mathrm{N}^{\circ} \\
\text { Muestra }\end{array}$ & DESIGNACION \\
\hline \multirow{11}{*}{ COLGADURAS } & \multirow{7}{*}{ CE088889 } & 1 & Cenefa exterior \\
\hline & & 2 & Hilo de la cenefa exterior, urdimbre \\
\hline & & 3 & Hilo de la cenefa exterior, trama \\
\hline & & 4 & Hilo de la cenefa exterior, urdimbre \\
\hline & & 5 & Centro \\
\hline & & 6 & Urdimbre del centro de la colgadura \\
\hline & & 7 & Trama del centro de la colgadura \\
\hline & CE088460 & 23 & Fragmento verde oscuro, en el interior del dobladillo \\
\hline & \multirow{3}{*}{ CE088478 } & 30 & Adhesivo sobre motivos \\
\hline & & 31 & Azul (parte central) \\
\hline & & 32 & Pardo parte inferior zona derecha (cenefa) \\
\hline
\end{tabular}




\section{Resultados}

Los análisis realizados mediante la aplicación de las diferentes técnicas analíticas pretenden alcanzar los siguientes objetivos. El primero de ellos es determinar la técnica de elaboración de los plisados y el segundo intentar explicar la aplicación del color en los estampados del resto de piezas textiles (colgaduras, indumentaria y tejidos planos).

\section{Indumentaria}

-Vestidos

- Delphos.- La identificación de las fibras del rojo y amarillo dio como resultado filamentos finos uniformes, de estructura interna invisible característicos de la seda. En el plisado beige, se identificaron además de las fibras correspondientes a la seda, otras con escamas propias de la lana (figura 5). En el análisis por TLC del Delphos rojo se identificó ácido carmínico, principal componente de un tinte de cochinilla.

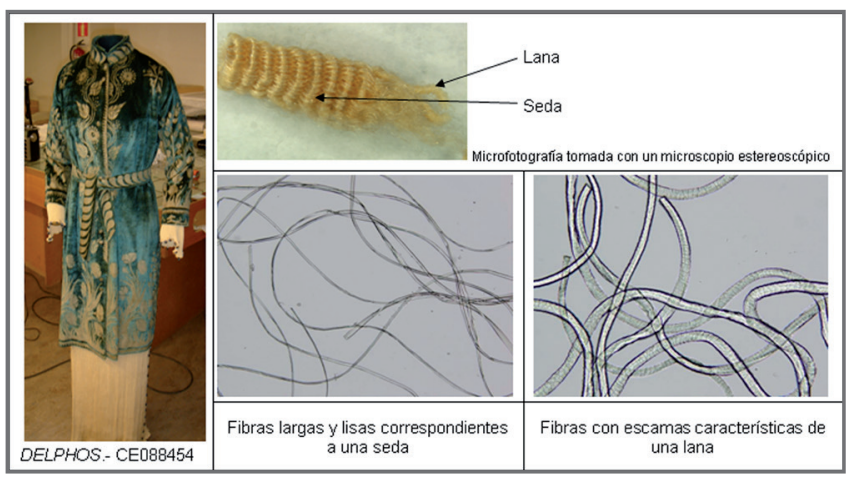

Figura 5.- Análisis de fibras del Delphos CE088454.

Dado que la naturaleza de la fibra es proteica, se optó de manera preliminar por realizar métodos microquímicos, como el test de almidón $\left(\mathrm{KI} / \mathrm{I}_{2}\right)$ y la tinción con fuschina. El resultado del primero de ellos fue negativo, es decir, no se detecta la presencia de almidón en las fibras. Mientras que el ensayo de coloración con fuschina que determina la presencia de algún tipo de recubrimiento proteico fue positivo. Obtenido este resultado, y para identificar el tipo de proteína se aplicó la cromatografía líquida de alta resolución. Dado que la fibra de seda es también proteica, el análisis no pudo identificar la presencia de albúmina u otro tipo de recubrimiento, aunque sí descartar la existencia de una cola, al no detectarse hidroxiprolina, aminoácido presente en este tipo de material.

- Vestido Eleonora.- De terciopelo negro y forrado en seda, con polvos metálicos plateados sobre las fibras. EI cromatograma de HPLC indica un aglutinante de huevo para las zonas metálicas.
- Vestido.- Es un vestido largo, sin mangas confeccionado en seda con polvos metálicos de latón y aluminio para los dorados y plateados respectivamente, y trazas de bermellón en las zona dorada. En el espectro EDX podemos ver elementos como el mercurio característico del bermellón, o el cobre y zinc pertenecientes al latón [figura 6].

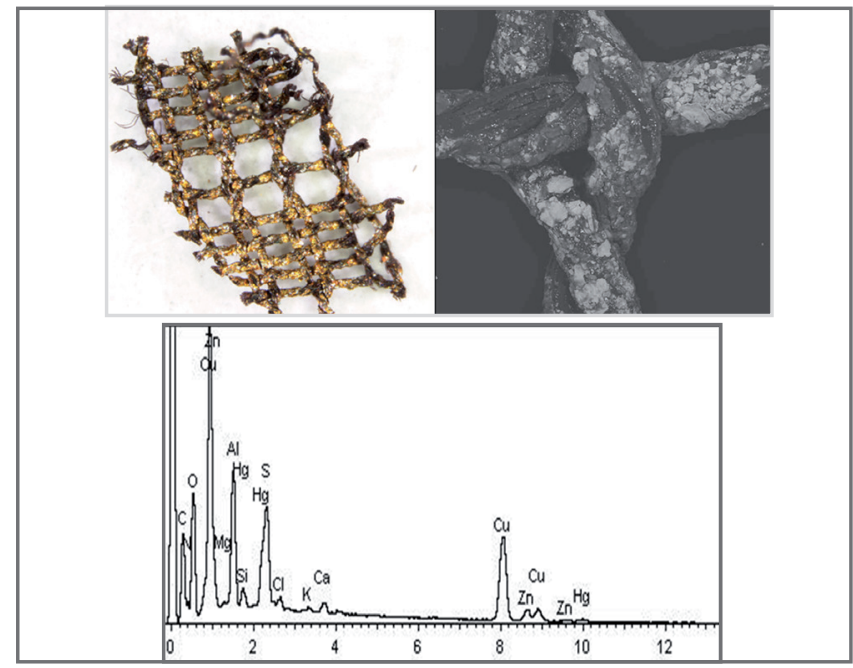

Figura 6.- Imágenes de microscopía óptica, electrones retrodispersados y espectro EDX del vestido CE 088405.

—Dalmática.- Realizada con seda en tonos verdes y azules, presenta polvos metálicos dorados y plateados. Los polvos metálicos al igual que en otros tejidos son de latón (para los dorados) y de aluminio (para los plateados). En la sección longitudinal de las fibras, se visualizan las fibras largas y lisas características de la seda. El test de indigotina resultó positivo para estas fibras, donde se aprecia el depósito del tinte azul de índigo o hierba pastel [figura7]. Con este test no puede distinguirse si el tinte está elaborado con índigo (Indigofera sp) o hierba pastel (Isatis Tinctoria $L$ ) puesto que sus diferencias de composición no son significativas y varían en función de cada especiey las condiciones de preparación del tinte [Cardon, 2007].

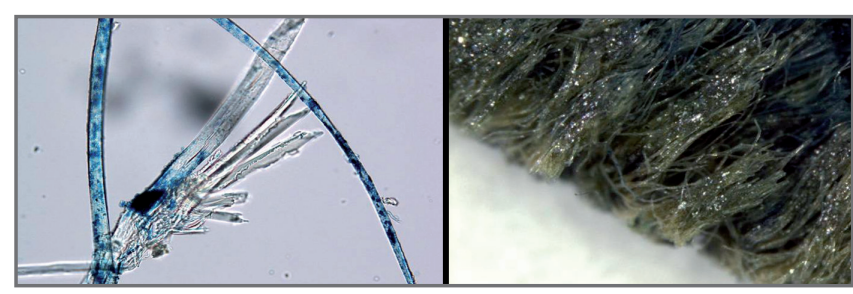

Figura 7.- Sección longitudinal de las fibras analizadas en la Dalmática CE088381.

- Chaqueta de teatro.- Confeccionada en seda y algodón con polvos metálicos de latón y aluminio en la seda.

-Túnica.- Indumentaria realizada en lana y seda con dorados de latón y plateados de aluminio. El colorante fue identificado por TLC como ácido carmínico, propio de un tinte cochinilla (Figura 8a). Bajo las zonas doradas se observa una capa de color blanquecino que se separó para realizar su análisis por HPLC dando como resultado huevo (Figura 8b). 


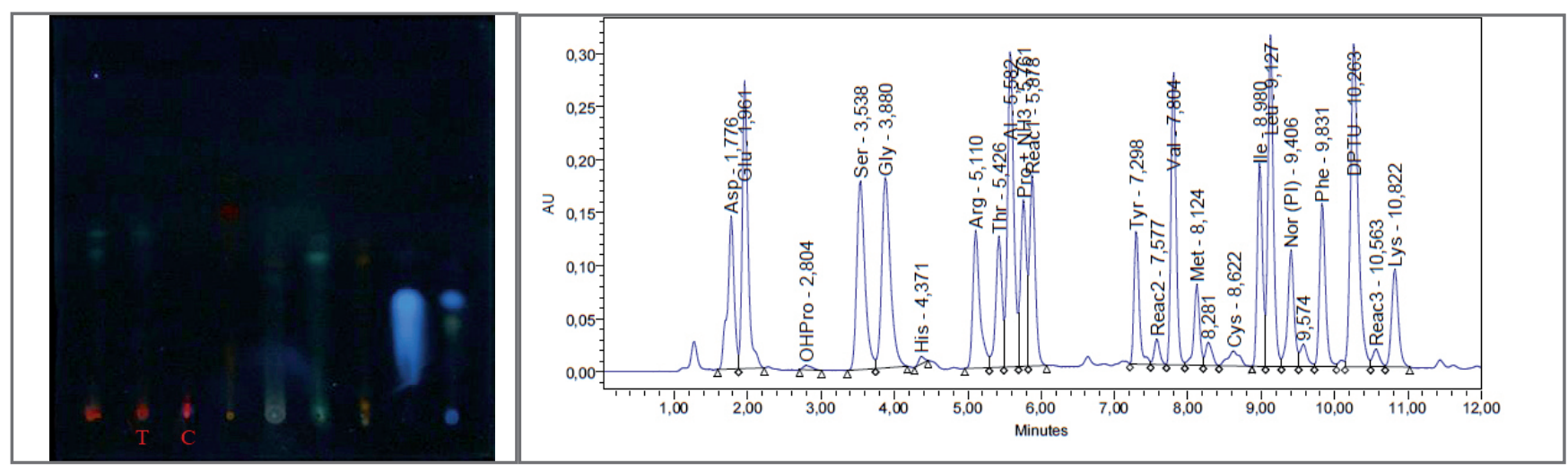

Figura 8.- Imagen superior: placa TLC correspondiente al colorante rojo de la túnica (T) y a diferentes patrones rojos, entre ellos el de cochinilla (C. Imagen inferior: Cromatograma de HPLC de la Túnica CE088389)

\section{Textiles}

\section{-Tejidos planos}

Fortuny diseñó un sistema que patentó en 1909 y que bautizó con el nombre de "procedimiento de impresión polícroma sobre tejidos y papel", en un intento de lograr la yuxtaposición correcta de los colores en el dibujo. Los tejidos denominados en el catálogo del CIPE como planos, tienen una gran variedad de estampados y en todos ellos se analizó la naturaleza de las fibras que los componen. En algunos casos se determina también la naturaleza de los pigmentos que dan la coloración, los elementos metálicos que proporcionan los efectos de oro o plata, e incluso el aglutinante usado para tan fin.

- Tejido CE088493.- Pieza de fibras de lino y algodón sobre fondo violeta, con repetición de motivos formados por una red de roleos pintados con un pigmento verde de arsénico y cobre (posible verde de Scheele), coronados en dorado con polvos metálicos de latón, aluminio y trazas de bermellón, aglutinados con huevo. El color violeta no pudo determinarse debido a la poca cantidad de muestra, pero en el espectro EDX del análisis SEM-EDX se detecta un alto contenido en hierro, aluminio, azufre y potasio, que podría indicar la presencia de azul de Prusia.

- Tejido CE088494.- Confeccionado con algodón gris con secuencias de escritura árabe en plateado que consigue mediante polvos de aluminio aglutinados con huevo. Tiene diferentes degradaciones cromáticas, no encontrándose ningún elemento de naturaleza inorgánica responsable del color del fondo.

- Tejido CE088517.- Pieza elaborada sobre fondo pardo de terciopelo de algodón, donde se distribuye una decoración cromática de amarillo claro, verde (de cobre) y dorado (de latón).

- Tejido CE088580.- Tejido elaborado en lino en dos colores. El rojo está realizado con bermellón mientras que el azul ha resultado ser una mezcla de indigotina, principal componente del tinte azul de índigo y azul de Prusia [figura9].

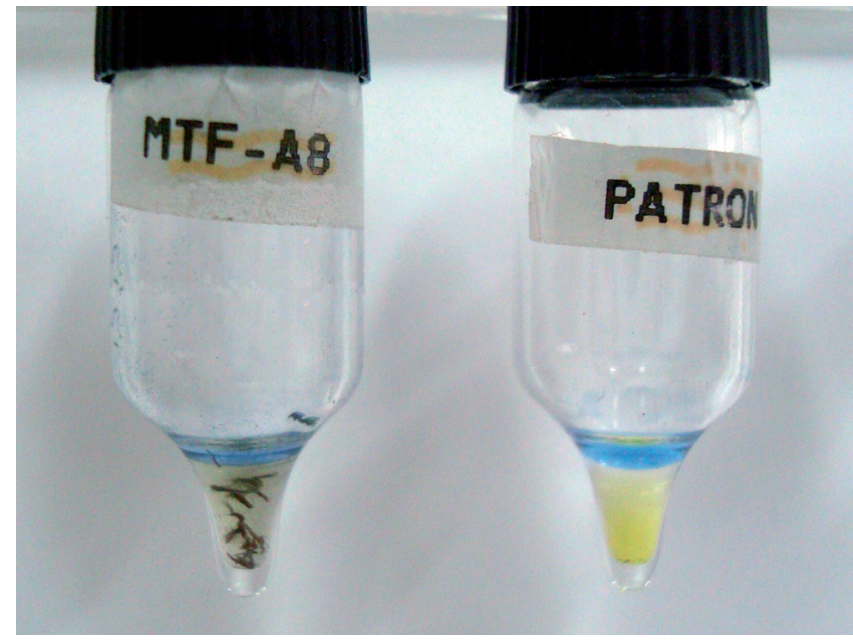

Figura 9.- Test de Indigotina para el azul del tejido CE088580

\section{- Colgaduras}

Las colgaduras están compuestas por diferentes materiales. Una de ellas, la primera de color verde y amarillo, está confeccionada en algodón y seda. La colgadura de color negro está realizada en algodón, mientras que la de color azul y beige es de seda.

El color verde de la primera colgadura parece estar ejecutado con una mezcla de tintes (en este caso amarillo y azul). El test para la identificación de indigotina fue positivo, confirmándose la existencia de un tinte azul, mientras que el tinte amarillo no pudo ser determinado.

Las colgaduras de color negro y azul presentan polvos dorados de latón (aleación de cobre y zinc) y de aluminio en los plateados [figura 10]. Tanto para el color negro de la primera colgadura como para el azul de la segunda no se ha determinado ningún pigmento inorgánico que identifique estos colores. Lo que hace suponer que sean de naturaleza orgánica. En el análisis por SEM-EDX del color azul se ha identificado cantidades apreciables de bermellón, muy molido y baritina o blanco fijo. Este último compuesto es 
casi transparente al mezclarlo con aceite, recomendado para acuarelas y frescos, es utilizado como carga y sustrato de los pigmentos laca. El efecto tornasolado del tejido, se debe probablemente a los granos dispersados del bermellón sobre el azul [figura 11].

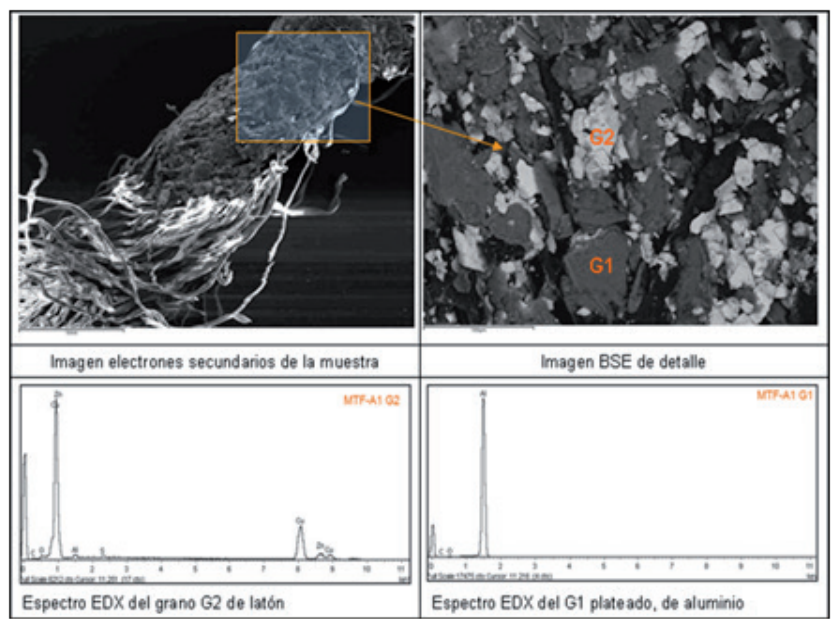

Figura 10.- Análisis por SEM-EDX de un fragmento de la colgadura negra CE088889

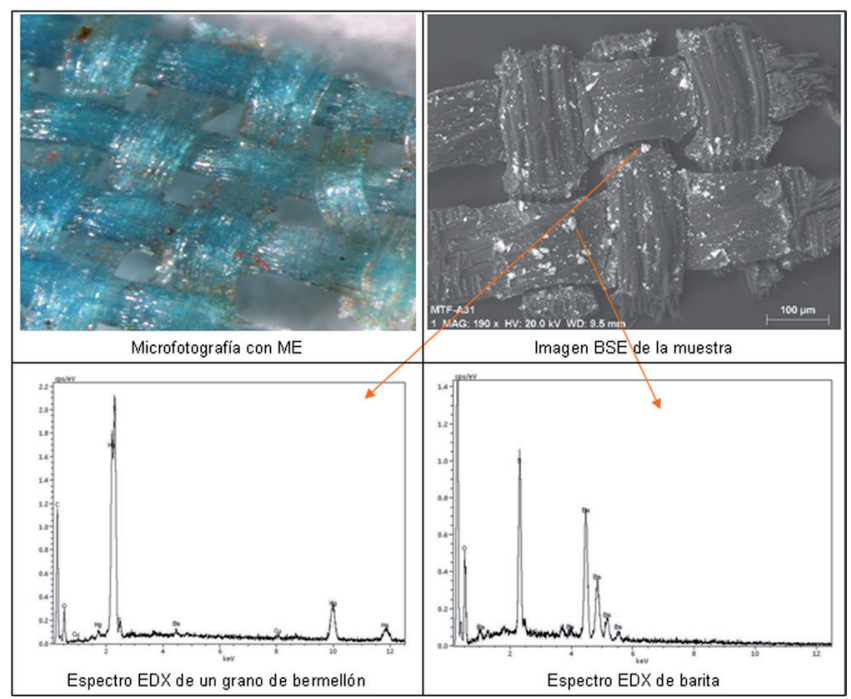

Figura 11.- Análisis por SEM-EDX del color azul de la colgadura CE088478

\section{Discusión de los resultados}

Uno de los aspectos más llamativos de los Delphos, es su peculiar plisado-ondulado de la tela. El ensayo de coloración con fuschina, para detectar proteína dio positivo. Se realizó también el test de almidón $\left(\mathrm{KI} / \mathrm{I}_{2}\right)$ cuya reacción fue negativa, pero se puso de manifiesto por las microfotografías de las fibras la existencia un recubrimiento amarillento en ellas. Los análisis por HPLC excluyen la presencia de una cola al no detectar la hidroxiprolina, pero no pudieron extraerse resultados concluyentes para la discriminación de otro material dada la naturaleza proteica de la fibra de seda.
Se identificaron colorantes unas veces aplicados como tintes, tal es el caso del Delphos rojo y la Túnica, y otros empleados como capa pictórica (como en algunos de los tejidos planos). El responsable del color rojo utilizado en el Delphos y la Túnica resultó ser ácido carmínico, propio de un tinte de cochinilla. No fue hasta 1891 cuando se consiguió la primera síntesis del ácido carmínico [Allevi, et ali. 1991], por lo que no podemos definir si su naturaleza es natural o sintética.

La presencia de indigotina principal compuesto del tinte de índigo o hierba pastel, se detectó en piezas como la Dalmática, y en los Tejidos planos CE08888460 y CE088580. El índigo fue uno de los últimos colorantes en los que se consiguió aclarar su composición química, Adolf Von Baeyer anunció la estructura química de la indigotina en 1883. A partir de 1897, se inicia el proceso de fabricación y comercialización para el índigo sintético. Por todo ello, no podemos afirmar que la naturaleza de este colorante azul sea natural o sintética. El color verde del Tejido plano CE088460 es una mezcla de índigo o hierba pastel con un colorante amarillo que no ha podido determinarse.

Las zonas doradas y plateadas, están realizadas con latón (aleación de cobre y zinc) para las primeras y con aluminio en las segundas. En el caso de los dorados se repite la utilización de pequeñas cantidades de bermellón muy molido, quizás para darle el aspecto tornasolado que presenta. La técnica de aplicación de estas escamas metálicas sobre el tejido, puede ser la de suspenderlas en huevo o la de aplicar primero este aglutinante $y$, posteriormente los polvos metálicos. Los análisis por HPLC no permiten discriminar entre yema y clara del huevo, al no mostrar ninguna diferencia cualitativa de los aminoácidos presentes en estos dos componentes.

La mayor parte de la decoración cromática de las piezas textiles se realizó con pigmentos de naturaleza inorgánica. Entre ellos, pigmentos verdes de cobre (tejido plano CE088517), de cobre y arsénico (posible verde de Scheele) del tejido plano CE088493 o rojos de bermellón tejido plano CE088580. En éste último tejido los análisis indican que la capa azul ha sido elaborada con una mezcla del tinte de índigo o hierba pastel y del pigmento inorgánico de azul de Prusia. Comparando el espectro de dispersión de energía de rayos $X$ (EDX) de esta muestra con los correspondientes al tejido plano CE088493 de color violeta, se observó que ambas capas tienen un espectro EDX y una morfología similar. Dada la poca cantidad de esta muestra violácea no pudo confirmarse la presencia del azul de Prusia o del índigo, pero la existencia del hierro en sus espectros EDX, y su similar morfología con el tejido CE088580 nos hace suponer que este color esté elaborado con una mezcla del tinte de índigo o hierba pastel y azul de Prusia.

Es importante indicar, que la poca cantidad de capa pictórica presente en las muestras extraídas de algunas de las piezas textiles, no facilitó la identificación de los aglutinantes de varios de los pigmentos hallados. 


\section{Conclusiones}

Aunque comparado por muchos de sus admiradores con Leonardo da Vinci, su país natal no parece darle toda la importancia que tuvo Fortuny en la historia. Genio inimitable, científico y artista utilizó pigmentos, metales y tintes unidos a materiales nobles y técnicas tradicionales que originaron formas y tonalidades nunca vistas en su época. Su doble formación hizo que experimentara con todo tipo de técnicas y materiales creando y patentando muchos de inventos. Revolucionario también de la moda y las artes escénicas, uno de sus grandes descubrimientos es el vestido Delphos cuyo plisado a día de hoy todavía no puede ser reproducido con exactitud. Esto último es debido probablemente por la existencia de un compuesto orgánico perdurable en el tiempo y añadido durante el proceso de fabricación que aunque en este estudio no ha podido ser identificado sí se ha puesto de manifiesto.

Con este artículo esperamos haber contribuido a un mayor conocimiento sobre la elaboración de tejidos e indumentarias de este fantástico creador e innovador del mundo de la moda.

\section{Agradecimientos}

A Ángela Arteaga por los análisis de TLC .A Monste Algueró, Ana Albar y Pedro Pablo Pérez por los análisis SEM-EDX.

\section{Bibliografía}

DE OSMA, G. (1994). Fortuny: The Life and Work of Mariano Fortuny. Editorial: Aurum Press

VV.AA. (2010). Inspiraciones. Mariano Fortuny y Madrazo. Madrid, Subdirección General de Promoción de las Bellas Artes. Ministerio de Cultura.

VV.AA. (2010) Fortuny: el mago de Venecia. Barcelona: obra Social de Caixa Catalunya.

VV.AA. (2012). Fortuny y Madrazo: an artistic legacy. New York: Queen Sofía Spanish Institute.

MATTHEWS, M (1948). Textile Fibers, John Wiley \& Sons, New York, pp 425.

GONZÁLEZ, E et ali. (2010).Proceso de intervención de un conjunto de siete piezas de indumentaria oriental: tratamiento de conservación-restauración y análisis de materiales constitutivos, Publicaciones del IPCE-Monografías: Investigación y Conservación de obras de arte oriental del Museo de Artes Decorativas, pp 42-69.

FERREIRA, E. et ali. (2004). The natural constituents of historical textile dyes, Chem. Soc. Rev, 33, pp 329.
GARCÍA, M.A.; CHÉRCOLES, R.; SANZ, E (2008). “Métodos analíticos desarrollados en el IPCE para el estudio de bienes culturales basados en la Espectroscopia de Infrarrojos por Transformada de Fourier y Técnicas Cromatográficas", La Ciencia y el Arte II. Instituto del Patrimonio Cultural de España. Ministerio de Cultura, pp 44-58

ARTEAGA, A., BORREGO, P., MORENO, M.,Platero,A (2009). "Tejidos del Valle del Nilo del Museo Arqueológico Nacional". Patrimonio cultural de España 1, pp 279-289.

CARDON, D (2007). "Natural Dyes: sources, tradition, technology and science", London: Archetype Publications Ltd.

SIMÓN SERFATY, J.L:.(1999). Atlas de fibras, árboles, arbustos y matorrales, Ministerio de Agricultura Pesca y Alimentación, pp 173.

HOFENK DE GRAAFF (2004). The Colourful Past. Origin, Chemistry and Identification of Natural Dyestuff, Switzerland: Abegg-Stiftung; London: Archetype Publications Ltd.

CARDON D (2007). Natural Dyes: Sources, tradition, technology and science, London: Archetype Publications Ltd.

ALLEVI, P et ali. (1991). The 1st Total Synthesis of Carminic Acid. Journal of the Chemical Society-Chemical Communications 18, pp 1319-1320

LLORENTE, L. (2005). Tejido plano de Fortuny. Octubre: modelo del mes, Museo del traje CIPE. 


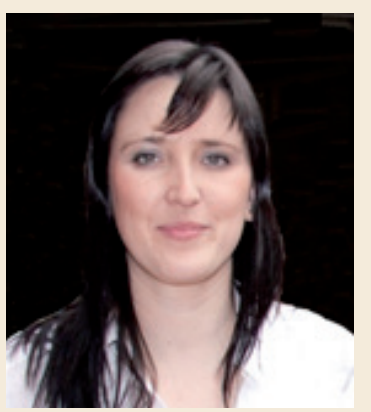

\section{Elena González Arteaga}

analisis.materiales.ipce@mecd.es; elenagoart@gmail.com Instituto del Patrimonio Cultural de España. Ministerio de Educación,Cultura y Deporte

Licenciada en Ciencias Químicas por la Universidad de Córdoba. Técnica del equipo multidisciplinar del Área de laboratorios de la sección de Análisis de Materiales del I.P.C.E, desde junio de 2006 realizando estudio de técnicas instrumentales aplicadas a la Investigación del Patrimonio Histórico. Química en el proyecto titulado "Evaluación de la idoneidad y riesgos potenciales en los procedimientos de limpieza sobre pintura y policromía: jabones de resina y ácidos biliares", del PNIC. Responsable de la parte técnica de análisis y difusión de dos proyectos europeos, el primero dentro del $7 \circ$ programa marco de la Unión europea NanoforArt finalizado, y del proyecto NanoRestArt en vigor actualmente y enmarcado dentro de Horizonte 2020.Ambos proyectos versan sobre la aplicación de nanopartículas a Patrimonio Cultural. Esta experiencia se refleja en diversos artículos y publicaciones.

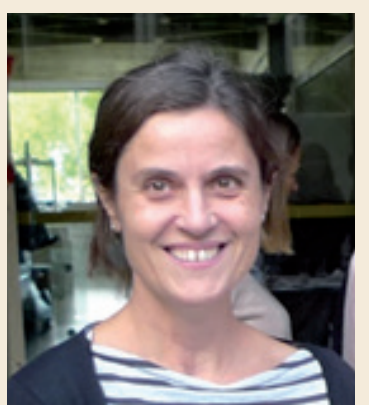

\section{María Antonia García Rodríguez}

antonia.garcia.r@mecd.es

Instituto del Patrimonio Cultural de España. Ministerio de Educación,Cultura y Deporte

Licenciada en Ciencias Químicas por la Universidad Complutense de Madrid. Desde 1992 hasta 1997, desarrolló su labor profesional en el Laboratorio de Control de Dopaje de Madrid (Consejo Superior de Deportes). En 1998 y 1999 colaboró en el Laboratorio de Salud Pública de la Comunidad de Madrid. Entre 2001 y 2005 trabajó realizando una asistencia técnica en el estudio de técnicas instrumentales aplicadas a la Investigación y Documentación del Patrimonio Histórico en el Instituto del Patrimonio Cultural de España (IPCE), donde actualmente, desde 2006, está como Titulada Superior en el Área de Investigación y Formación. Su labor consiste en el estudio relacionado con la pintura mural y material arqueológico, así como el análisis de materiales orgánicos en otro tipo de obras de arte. La mayoría de los cursos realizados o participación en diversas jornadas, se centran en el desarrollo de nuevas técnicas analíticas y cursos entorno a la conservación del Patrimonio, entre otros. Es autora de varios artículos que aparecen en diversas publicaciones.

\section{Artículo enviado el 14/11/2017}

Artículo aceptado el 04/12/2017 